Meta

Journal des traducteurs

Translators' Journal

\title{
La traduction : histoire et théorie
}

\section{Paul St-Pierre}

Volume 35, numéro 1, mars 1990

Actes du colloque international " La traduction proligère "

URI : https://id.erudit.org/iderudit/004298ar

DOI : https://doi.org/10.7202/004298ar

Aller au sommaire du numéro

Éditeur(s)

Les Presses de l'Université de Montréal

ISSN

0026-0452 (imprimé)

1492-1421 (numérique)

Découvrir la revue

Citer cet article

St-Pierre, P. (1990). La traduction : histoire et théorie. Meta, 35(1), 119-125.

https://doi.org/10.7202/004298ar d'utilisation que vous pouvez consulter en ligne.

https://apropos.erudit.org/fr/usagers/politique-dutilisation/ 


\section{LA TRADUCTION: HISTOIRE ET THÉORIE}

PAUl ST-PIERRE

Université Laval, Québec, Canada

Face au texte à traduire le traducteur hésite : suffit-il d'en abstraire l'esprit ou doit-il le reproduire jusque dans sa littéralité, faut-il y être scrupuleusement fidèle ou peut-il l'adapter librement au nouveau contexte et à la nouvelle langue ? Ces apories fondamentales - l'esprit ou la lettre, fidélité ou adaptation -, apories dont le contenu précis est toujours déterminé contextuellement ${ }^{2}$, conditionnent la pratique du traducteur. Devant l'impossibilité d'une correspondance absolue entre la traduction et le texte original correspondance impossible mais néanmoins ardemment recherchée ${ }^{3}$ - le traducteur doit faire des choix, et ses choix l'obligeront à se situer face au texte original, à prendre position, à perdre cette transparence qu'on lui souhaite tant $t^{4}$, situation d'autant plus inconfortable pour le traducteur que ses choix ne seront nullement fonction du texte qu'il traduit, même si pour se justifier il prétendra souvent le contraire. En effet, ce n'est pas le texte original qui va déterminer que la traduction devra être fidèle, que c'est l'esprit et non la lettre qui devra être respecté, qui va dicter au traducteur d'adapter le texte à la langue d'arrivée ou, à l'inverse, de maintenir les structures de la langue de départ. Ainsi, un même texte suscitera des approches différentes, contradictoires même, comme le reconnaît C.-J. Panckoucke dans la préface de sa traduction du Jérusalem délivrée du Tasse (1785: 5): «Nous avons dans notre langue deux traductions estimées de la Jérusalem; celle de Mirabaud, et celle de $\mathbf{M}$. le $\mathbf{B}^{* * *}$. Toutes les deux ont été réimprimées plusieurs fois : cependant ces deux traductions sont si différentes l'une de l'autre, qu'elles semblent prouver qu'on n'est pas encore bien d'accord parmi nous sur la meilleure manière de traduire, et surtout de traduire les Poètes.» Et il poursuit (pp. 11-12) : «Je me suis proposé un but tout différent de ces deux traducteurs: j'aurais voulu faire une traduction littérale et fidèle, qui pût être imprimée à côté du texte, qui ne fût destinée qu'à cet usage, qui rendît le génie du Tasse, et qui servît en même temps à faciliter la lecture et l'intelligence de ce grand Poète.» Ainsi, aux deux traductions mentionnées vient s'ajouter une troisième, qui se distingue encore de celles-ci. Or, les différences entre ces trois versions du poème du Tasse ne sont pas purement accidentelles; elles tiennent plutôt au rôle joué par les critères contextuels dans la transformation qu'opère la traduction. La version de Panckoucke sera différente et aura donc droit de cité, du fait que le traducteur vise un but autre que celui que se donnaient Mirabaud et M. le $B^{* * *}$, c'est-à-dire la littéralité. Et ce n'est pas seulement dans la traduction de textes particuliers que les approches peuvent diverger; il peut en aller de même pour tout un genre. Prenons l'exemple de la poésie. En préface à sa traduction des satires de Perse en 1771, l'abbé Le Monnier explique sa décision de produire une traduction servile (p. xiii): «Un traducteur, et sur-tout le traducteur d'un poëte, doit se comporter comme ces députés qui étoient tenus de répéter les mots qui leur avaient été dictés avant leur départ.» Un an plus tard, Vauvilliers prendra le contre-pied dans son Essai sur Pindare (1772: 27): «J'observerai seulement, que si on peut se flatter de rendre un Philosophe, un Historien, et même quelquefois un Orateur, par ce qu'on appelle une traduction littérale, c'est-à-dire servilement attachée à la valeur précise de chaque mot, et à l'ordre de construction; c'est assurément une prétention chimérique quand on entreprend 
de faire passer dans une autre langue les sublimes accents de la Poésie la plus noble et la plus hardie (...)» Dans un cas, la poésie exigerait la littéralité, dans l'autre, elle la bannirait. Et pour que l'on n'attribue pas l'existence de contradictions à un certain sousdéveloppement de la théorie de la traduction au dix-huitième siècle, citons brièvement quelques exemples du nôtre.

Plutôt que de se résorber, les contradictions se multiplient, notamment en ce qui concerne la définition du sens, c'est-à-dire de ce qui est considéré le plus souvent constituer l'objet même de la traduction. Ainsi, dans Interpréter pour traduire, Marianne Lederer prend position contre ceux qui situeraient le sens au plan des structures linguistiques pour insister sur sa nature discursive. Pour elle, la tâche du traducteur (Seleskovitch et Lederer, 1986: 25-26) «ne consiste pas à transposer les faits observables de la langue ni à mettre au point des clefs de conversion entre les langues mais à restituer le contenu du message». On reconnaît ici la conception de la traduction qu'avait saint Jérôme - «c'est le sens qu'il faut rendre, et tout le sens, mais non les mots ${ }^{5} »-$, une conception taxée d'idéaliste par Henri Meschonnic puisqu'elle présupposerait la possibilité de séparer le sens des mots, le signifié du signifiant ${ }^{6}$. De même, la définition de la traduction en fonction de la communication ${ }^{7}$ ne fait pas l'unanimité. Dans La Tâche du traducteur, Walter Benjamin $(1971: 261)$ note «qu'[u]ne traduction cependant, qui veut communiquer, ne saurait transmettre que la communication - donc quelque chose d'inessentiel. Et c'est là, aussi bien, l'un des signes auxquels se reconnaît la mauvaise traduction». Benjamin, il est vrai, traite ici de la traduction littéraire, mais cela n'a pas nécessairement pour effet de réduire la généralité de son propos, puisque la distinction entre le littéraire et le non-littéraire n'est pas elle-même sans poser problème. Comme le remarque George Steiner dans Après Babel (1978:229): «Les attaques lancées contre la traduction de textes poétiques ne sont que l'expression la plus radicale de la thèse commune selon laquelle on ne traduit pas une langue sans un déchet essentiel.»

Ces quelques exemples permettent donc de constater que les traducteurs se retrouvent souvent en contradiction, non seulement par les différentes traductions qu'ils donnent d'un texte original mais aussi au plan des principes régissant leur pratique. Or, j'insiste, ces contradictions sont de l'essence même de la traduction et ne doivent pas comme c'est trop souvent le cas - être gommées par un discours normatif qui tente de les réduire en prescrivant une seule façon de traduire, que ce soit littéralement, librement, fidèlement, servilement, ou autrement encore. Au contraire, ces contradictions doivent être maintenues, car ce sont précisément elles qui nous renseignent sur les choix ouverts au traducteur, sur les critères utilisés, sur les valeurs en cours. Une théorie de la traduction aura donc à identifier pour un contexte donné les formes d'équivalences possibles en interrogeant non pas les textes mais leur modalisation, la prise en charge du texte de départ par une visée qui lui est extérieure mais en même temps déterminante. C'est dire qu'une théorie de la traduction devra tenir compte de l'histoire.

En France, entre les seizième et dix-huitième siècles, les approches des traducteurs de même que le rôle attribué à la traduction ont changé. Tandis qu'au seizième siècle la traduction avait une fonction essentiellement novatrice, par son importation dans la langue vulgaire de mots nouveaux, d'idées et de formes nouvelles, deux cents ans plus tard elle servait surtout à apporter la confirmation de la supériorité de la langue et de la nation françaises. Le développement de la France, notamment sous Louis XIV, avec l'institutionnalisation progressive de la culture mise au service de l'État, a modifié le statut et la fonction des traductions, ce qui se vérifie dans les buts que se fixent les traducteurs et dans les moyens qu'ils se donnent pour les atteindre. Au début du seizième siècle, les traducteurs ont porté beaucoup d'attention à la langue, en insistant sur sa pauvreté relative, sur son insuffisance à exprimer le monde. Face aux langues classiques, et même aux 
autres langues vulgaires (l'espagnol et l'italien en particulier), les traducteurs se sont donné comme but premier l'enrichissement et le perfectionnement du français. Dans son épître dédicatoire à La Manière de bien traduire d'une langue en aultre, Étienne Dolet (1540:4-5) écrit: «Car je scay, que quand on voulut reduire la langue Grecque, et Latine en art, cela ne fut absolu par ung homme, mais par plusieurs. Ce qui se faira pareillement en la langue françoyse: et peu à peu par le moyen, et travail des gens doctes elle pourra estre reduicte en telle parfection, que les langues dessusdictes. A ceste cause (Seigneur tout humain) je te requiers de prendre ce mien labeur en gré, et s'il ne reforme totallement nostre langue, pour le moyns pense, que c'est commencement, qui pourra parvenir à fin telle, que les estrangiers ne nous appelleront plus Barbares.» Au dix-huitième siècle, par contre, le français a un tout autre statut. L'abbé Des Fontaines souligne cette évolution (1743: xvi-xvii): «Si notre langue étoit aussi grossière, aussi rude, aussi foible qu'elle étoit autrefois, j'avoue qu'elle seroit peu capable de faire honneur aux Anciens. Mais elle est devenue si douce, si harmonieuse, si régulière, si expressive, qu'elle pourroit presque être mise en paralléle avec les belles langues de l'antiquitée ${ }^{8}$.» Au dix-huitième siècle, c'est la conformité des œuvres étrangères aux critères nationaux de goût, aux bienséances, à ce qui est français, qui intéresse les traducteurs. Ainsi, un des débats auquel ils participeront tout au long du siècle sera de savoir si une traduction doit se lire comme une œuvre française ou au contraire porter les marques de son origine étrangère. Le traducteur anonyme des Euvres complètes d'Alexandre Pope écrira (1779: 186): «Ce qui vient des Étrangers, dit Aristote dans sa Rhétorique, paroît admirable, plaît et réjouit. Cette pensée, si elle est vraie, ne peut trouver son application parmi nous. Soit amour de Nation, ou, comme il nous plaît de l'appeller, amour du bon Goût, on nous accuse de vouloir tout ramener au nôtre; et il faut avouer que l'air étranger, loin de nous plaire, est souvent un fâcheux préjugé contre tout ce qui en porte le caractère.» Dans l'espace de deux siècles, donc, l'incertitude s'est transformée en triomphalisme.

Pour comprendre ce changement, il est nécessaire de mettre le développement de la traduction en rapport avec celui d'autres domaines. On peut ici en mentionner deux, d'une grande importance pour la traduction : la langue et l'imprimerie. Pour ce qui est du premier, cette période se caractérise par une pléthore de textes sur la rhétorique, sur la grammaire et l'orthographe du français, par de nombreuses défenses et illustrations de la langue. En plus, une série de mesures officielles promeut l'utilisation du français. En 1539 , l'ordonnance de Villers-Cotterêts rend générale l'utilisation du français dans tous les actes administratifs. En 1624, on admet, même si ce n'est qu'exceptionnellement, que les soutenances de thèse aient lieu en langue vulgaire. En 1676, il est décidé que les inscriptions sur les monuments élevés à Louis XIV seront en français et, quatre ans plus tard, le français est utilisé pour la première fois dans l'enseignement du droit. Encore plus importante est la création de l'Académie française en 1635, avec la mission de codifier la langue, ce qu'elle fait avant la fin du siècle en publiant son dictionnaire et en établissant les règles de l'orthographe. En même temps que le français se répand, la nation se construit, et sur les deux plans se produit une certaine uniformité. Par ailleurs, cette même volonté d'uniformité se voit dans l'extension de l'autorité royale sur l'imprimerie. Avec l'institution des privilèges royaux en 1563 , l'université perd le droit de censure aux dépens du pouvoir central. En 1624, le système de censure royale est mis en place, renforçant le pouvoir de l'autorité. D'autres mesures encore - en 1686, 1701, 1704 et 1723 notamment - vont dans le même sens. Ce n'est que vers la fin du dix-huitième siècle, avec l'affaiblissement du pouvoir du roi, qu'une certaine libéralisation se fait sentir. L'histoire de l'imprimerie sous l'ancien régime peut donc être considérée comme l'histoire d'une tentative de la part des autorités de contrôler à travers la production de livres la production de la culture et la définition de l'identité nationale. 
Dans ce contexte, la traduction avait un rôle très important à jouer puisque, en situant la culture nationale par rapport aux cultures étrangères, elle la définissait. L'évolution de la traduction - le changement dans les critères utilisés par les traducteurs - doit donc être compris en rapport avec les développements dans d'autres domaines. Dans sa transformation du texte original, le traducteur se sert de critères, et ces critères lui sont dictés non par le texte à traduire mais par son époque. Ce ne sera donc que par une approche historique qu'il sera possible de dire non seulement de quelle façon le texte a été traduit - quels ont été les critères utilisés - mais aussi pourquoi il a été traduit de cette façon; ce ne sera qu'alors que sera précisée la fonction accordée à la fois au traducteur et à la traduction.

De la même façon que les approches des traducteurs du seizième et du dix-huitième siècles doivent être situées en rapport avec les contextes où elles ont pu se développer, les différentes déterminations des théories de la traduction au vingtième siècle doivent être explicitées, afin que deviennent clairs les enjeux de la théorie de la traduction à notre époque. Dans l'impossibilité d'en faire une étude exhaustive ici, je ne soulignerai que deux aspects - l'institutionnalisation de la traduction et son expansion - par le biais d'une citation tirée de l'Introduction à Interpréter pour traduire. Marianne Lederer et Danica Seleskovitch écrivent (1986: 10-11):

La conception de la traduction élaborée à l'ESIT est le fruit de la réflexion de praticiens sur leur propre pratique. L'explication de l'opération de traduction a permis de mettre au point des méthodes d'enseignement qui font des diplômés de l'ESIT des traducteurs et des interprètes appréciés par l'ensemble du monde international.

Le français perdant progressivement son caractère de langue universelle, ces professionnels font œuvre de salut public dans un pays porté, du fait de sa longue hégémonie linguistique, à l'insouciance à l'égard de la traduction. Défendre aujourd'hui la langue française, c'est aussi offrir des relais fiables dans les vecteurs de la pensée que sont la traduction et l'interprétation.

Dans cette citation, les deux aspects sont clairement soulignés: d'une part, une théorie de la traduction élaborée dans le cadre institutionnel d'une école de traduction et, d'autre part, l'expansion prise par la traduction du fait que le français aurait perdu de son universalité. Examinons d'abord le deuxième aspect. Les auteures citées semblent vouloir donner un rôle quelque peu paradoxal à la traduction. Celle-ci viendrait en quelque sorte suppléer le français en donnant accès à une certaine universalité que celui-ci aurait désormais perdue, mais en même temps elle contribuerait à la défense de la langue nationale. Tout comme au dix-huitième siècle, le rôle de la traduction est à définir en rapport avec la langue nationale, mais tandis qu'au dix-huitième siècle le français avait acquis une universalité telle que l'on effaçait dans les textes traduits les marques de l'origine étrangère, au vingtième siècle traduire en français est devenu une obligation dictée par la nécessité de comprendre et non de dominer, du fait que le français n'a plus son rôle d'intermédiaire - de truchement - entre les nations. La transparence du français, qui lui venait de son hégémonie, est devenue opacité, rendant nécessaire la traduction. Et si la traduction crée toujours des hiérarchies ${ }^{9}$, ces hiérarchies restent toujours à définir. Au vingtième siècle, on est peut-être plus près de l'incertitude provoquée par les langues étrangères au seizième siècle que du triomphalisme du dix-huitième. Ainsi pourrait s'expliquer le développement à notre époque de la terminologie, à laquelle se porte une attention particulière en période de crise linguistique. Au seizième siècle, l'intérêt suscité par la traduction des termes est grand, tandis que les traducteurs du dix-huitième siècle ne sont guère intéressés par un tel sujet, étant donné qu'à cette époque c'est le texte étranger qui doit se plier au français et non l'inverse. Tout ce qui n'entrerait pas en français serait tout simplement à 
exclure, à éliminer. Au vingtième siècle, au moment même où la langue française perdrait son caractère de langue universelle, réapparaît un intérêt pour la terminologie, réapparition qui est peut-être le symptôme de l'incertitude provoquée par le renversement qui se produit dans la hiérarchie des langues.

L'autre élément à souligner ici est l'institutionnalisation de la traduction. Si les traducteurs du seizième siècle traduisent «à la dérobée», «en cachette», «pendant les loisirs» — la traduction étant une pratique inavouable et peu sérieuse —, le vingtième siècle est marqué par la création de cadres institutionnels et par la reconnaissance de la traduction comme métier. On fonde des écoles de traduction, crée des sociétés de traducteurs, publie des revues de traductologie, crée des services de traduction dans l'entreprise et au niveau gouvernemental; ce sont autant d'actes qui, en transformant les conditions dans lesquelles se fait la traduction, en changent aussi la nature.

L'institutionnalisation et l'expansion que connaît la traduction ne sont pas sans effet sur la théorisation de cette pratique. Dans l'extrait de Interpréter pour traduire déjà cité, l'insistance à souligner que la théorie proposée est le fruit de la réflexion de praticiens sur leur propre pratique a pour fonction de valider la théorie présentée. Une théorie, semblet-on affirmer, n'aurait de validité que dans la mesure où elle résulterait d'une pratique. Mais ce que l'on oublie de souligner, c'est la spécificité de toute pratique, c'est-à-dire le caractère particulier du lieu à partir duquel cette théorie a été élaborée, oubli que l'on peut caractériser comme idéologique. Qu'elle ait été élaborée dans le cadre institutionnel d'une école de traduction, qu'elle conduise à des méthodes d'enseignement n'est pas sans rapport avec le contenu même de la théorie, tout comme le fait que cette conception ait été développée à un moment où le français perdait son caractère universel, à un moment donc où il devenait nécessaire de traduire. Avec la professionnalisation de la traduction et le rôle qu'elle est appelée à jouer en raison du déclin du français, il devient impératif que ce qui constitue l'essentiel d'un texte puisse passer indemne d'une langue à une autre. Dans le premier cas, pour protéger le métier (de traducteur, mais aussi de professeur de traduction), dans le deuxième, pour protéger le français. Or, ce sont les structures linguistiques qui ne peuvent pas être traduites, du moins sans pertes évidentes. Il a donc fallu que l'objet de la traduction - le sens - se situe ailleurs que dans ces structures, car à la fois le métier et la nation demandent une pure traduisibilité, une traduisibilité sans déchets. Citons ici encore une fois Marianne Lederer (Seleskovitch et Lederer 1986: 25-26) : «Le sens qui intéresse la traduction n'est pas celui qui préoccupe le philosophe, et rarement celui dont s'empare l'artiste. Il n'est pas non plus celui qu'étudie la sémantique; celle-ci ne s'intéresse qu'aux significations de la langue, démarche fort légitime pour le linguiste, mais qui serait paradoxale pour le traducteur dont la tâche ne consiste pas à transposer les faits observables de la langue ni à mettre au point des clefs de conversion entre les langues mais à restituer le contenu du message.» Il y aurait donc au moins quatre sens différents, et celui du traducteur se limiterait au contenu du message, au vouloir-dire de l'auteur, à ce qui est censé pouvoir se traduire sans perte d'une langue à une autre ${ }^{10}$. L'objet de la traduction est donc défini ici en fonction du statut du traducteur, du rapport de la traduction à la langue nationale, de la nécessité de traduire, tout comme il l'était au seizième et au dix-huitième siècles. Il n'y a pas nécessairement erreur à définir ainsi l'objet de la traduction, en autant qu'est reconnue sa nature historique. Par contre, ne pas l'admettre empêche qu'il y ait théorie et condamne à rester dans l'idéologie. Comme le note Michel de Certeau dans L'opération historique (1974: 16): «La dénégation de la particularité du lieu étant le principe même de l'idéologie, elle exclut toute théorie.» $\mathrm{Ce}$ n'est qu'en élucidant ce lieu d'où l'on parle en tant que théoriciens de la traduction, d'où l'on traduit en tant que traducteurs, que se fera une théorie de la traduction, et non une idéologie. 
L'hésitation du traducteur devant le texte a donc un sens; elle fait transparaître son inquiétude face à l'obligation de choisir, sans autre autorité que la sienne propre et celle de son époque. Que le traducteur se limite à la reproduction ou, au contraire, qu'il opte pour la construction, son choix sera fonction non du texte à traduire mais du contexte dans lequel se fait la traduction, contexte qui définit, comme le note Annie Brisset (1989: 52), «les conditions d'exercice de la fonction translative». Or, cette hésitation est signe de la conscience qu'a le traducteur de la gravité de ses choix. Mais trop souvent il tentera d'effacer toute trace d'indécision, il essaiera de justifier ses décisions en faisant appel à des normes à la fois absolues et illusoires. L'hésitation sera peut-être abolie, du moins temporairement, mais à un prix trop élevé, car c'est l'idéologie qui règne alors. C'est cela que peut nous apprendre une approche historique à la traduction, qui permet alors que la traduction s'ouvre sur sa vérité.

\section{NOTES}

1. Ce travail se situe dans le contexte d'un projet de recherche sur l'histoire de la traduction en France, projet subventionné par le Conseil de recherche en sciences humaines du Canada.

2. La notion de «fidélitề, par exemple, peut recevoir des sens assez différents selon les contextes et selon les traducteurs. $\mathrm{Si}$, pour certains, traduire fidèlement signifie rendre l'expression même du texte original, pour d'autres, une traduction fidèle est celle qui se libère de l'expression pour serrer de plus près l'intention de l'auteur, le message du texte. Voir St-Pierre (1988) pour la discussion de la «fidélité» des traducteurs du seizième siècle français.

3. L'histoire de la traduction grecque de l'Ancien Testament (la Septante) est à cet égard exemplaire. Selon Aristée, les soixante-douze traducteurs ont travaillé seuls, chacun dans sa cellule, mais ont néanmoins produit une traduction unique. Or, cette histoire racontée par Aristée et répétée à maintes reprises depuis a une fonction qui est de créer une correspondance absolue, une correspondance miraculeuse, entre le texte et sa traduction. Pour une analyse plus approfondie de ces rapports entre texte et traduction, voir St-Pierre (1985).

4. Gin, dans la préface à sa traduction des CEuvres complètes d'Homère (1786: xiii), condamne la traduction en vers en ces termes: «(...) la mécanique de notre versification, si différente de celle des Grecs et des Latins, dénature essentiellement l'original qu'on s'efforce de représenter : elle fixe l'attention sur le traducteur, dont le plus grand mérite est de se faire oublier.»

5. Traduction de J.-P. Vinay (1968: 737).

6. Henri Meschonnic (1973: 319), Pour la poétique II. Voir aussi les travaux de Jacques Derrida, notamment pour ce qui est de l'impossibilité d'un pur signifié qui échapperait à la chaîne des signes.

7. Marianne Lederer, «Transcoder ou réexprimer?», Interpréter pour traduire (Seleskovitch et Lederer, 1986: 18) : «(...) le besoin de traduction découle directement du besoin de communication (...)»

8. André Dacier avait déjà exprimé de pareils sentiments en parlant d'une traduction par de Meziriac $(1721$ : xvi) : «(...) de son temps nostre Langue n'estoit pas dans toute la perfection où elle est aujourd'huy, et (...) elle n'avoit pas ces graces et ces délicatesses que les grands hommes, qui ont écrit dans nostre siècle, luy ont donné dans leurs ouvrages, et auxquelles on ne peut nier sans ingratitude et sans injustice que 1'Académie Françoise n'ait beaucoup contribué."?

9. À ce sujet, voir Vincente Refael, Contracting Colonialism: Transition and Christian Conversion in Tagalog Society under Early Spanish Rule, et James T. Siegal, Solo in the New Order: Language and Hierarchy in an Indonesian City.

10. Marianne Lederer, «Transcoder ou réexprimer?», Interpréter pour traduire (Seleskovitch et Lederer, $1986: 36)$ : «En fin de compte et sans chercher à être paradoxal, on serait tenté de dire que les langues sont extérieures au processus de la traduction; elles sont le réceptable du sens qui est exprimable dans n'importe laquelle d'entre elles; elles ne se confondent pas avec lui.»

\section{BIBLIOGRAPHIE}

ANONYME, trad. (1779): CEuvres complètes d'Alexandre Pope, Paris, Vve Duchesne.

BENJAMIN, Walter (1971) : «La tâche du traducteur», QEuvres, trad. Maurice de Gandillac, Paris, Denoël, I.

BRISSET, Annie (1989): «Sociocritique de la traduction. Un corpus québécois», Cahiers de recherche saciologique, XII, pp. 51-62.

CERTEAU, Michel de (1974) : «L'opération historique», Faire de l' histoire (sous la dir. de Jacques Le Goff et Pierre Nora), Paris, Gallimard. 
DACIER, André (1721): Les Vies des hommes illustres de Plutarque, Paris, Michel Clousier et al. DES FONTAINES, l'abbé, trad. (1743) : Les Euvres de Virgile, Paris, Guillau.

DOLET, Étienne (1540) : La Manière de bien traduire d'une langue en aultre, Lyon, Dolet.

GIN, trad. (1786): CEuvres complètes de Homère, Paris, Didot l'aîné.

LE MONNIER, l'abbé, trad. (1771) : Satires de Perse, Paris, Ch. Ant. Jombert et al.

MESCHONNIC, Henri (1973) : Pour la poétique II, Paris, Gallimard.

PANCKOUCKE, C.-J., trad. (1785) : Jérusalem délivrée, Paris, Hôtel de Thou et chez l'auteur.

RAFAEL, Vicente (1988): Contracting Colonialism : Transition and Christian Conversion in Tagalog Society under Early Spanish Rule, Ithaca, Cornell University Press.

SELESKOVITCH, Danica et Marianne LEDERER (1986) : Interpréter pour traduire, Paris, Didier Érudition.

SIEGAL, James T. (1986): Solo in the New Order: Language and Hierarchy in an Indonesian City, Princeton, Princeton University Press.

STEINER, George (1978) : Après Babel, trad. Lucienne Lotringer, Paris, Albin Michel.

ST-PIERRE, Paul (1985) : «Translation and Writing», Texte, no 4, pp. 223-233.

ST-PIERRE, Paul (1988) : «The Historical Nature of Translation», Communication présentée au Scandanavian Symposium on Translation Theory III, Oslo.

VAUVILLIERS, trad. (1772) : Essai sur Pindare, Paris, Paul-Denis Broca.

VINAY, J.-P. (1968) : «La Traduction humaine», Le Langage, Paris, Gallimard. 